\title{
ESTUDIO DE OPINIONES SOBRE EL DESEMPEÑO ECONÓMICO: EVIDENCIA PARA LOS PAÍSES DE AMÉRICA LATINA Y EL CARIBE ${ }^{\circ}$
}

\author{
STUDY OF INDIVIDUALS' OPINIONS ABOUT \\ ECONOMIC PERFORMANCE: EVIDENCE FROM LATIN \\ AMERICAN AND CARIBBEAN COUNTRIES
}

Martín Llada*

enviado: 16 febrero 2020 - aceptado: 12 julio 2020

\begin{abstract}
Resumen
Este trabajo estudia asociaciones entre opiniones sobre desempeño económico y flujos de información económica. Para 18 países de América Latina y el Caribe en el período 1995-2016, se encuentra 1) una asociación positiva entre un indicador de expectativas y el indicador de evaluaciones económicas esperadas, 2) controlando por las expectativas reportadas en el período t, información disponible en t- 1 factible de ser incorporada para formular expectativas - contribuye a explicar la evolución de las evaluación esperadas, 3) se evidencia instancias de sub y sobre-reacción y 4) no se evidencia heterogeneidad en las opiniones de individuos con diferentes características sociodemográficas e inclinación política. Los resultados 1 , 2 y 3 son inconsistentes con los postulados de los modelos FIRE (full-information rational expectation).
\end{abstract}

Palabras clave: opiniones, expectativas, sentimiento, macroeconomía. Códigos JEL: E7, E71.

Llada, M. (2021). Estudio de opiniones sobre el desempeño económico: evidencia para los países de América Latina y el Caribe. Estudios económicos, 38(76), 69-94.

* Universidad de Buenos Aires. Facultad de Ciencias Económicas y CONICET Universidad de Buenos Aires. Instituto Interdisciplinario de Economía Política de Buenos Aires. Buenos Aires, Argentina. Correo electrónico: 1ladamartin@gmail.com. ORCID: https://orcid.org/0000-0002-7526-8816 


\begin{abstract}
This paper studies associations between the individuals' opinions about economic performance and economic information flows. For 18 Latin American and Caribbean countries for the period 1995-2016, we find: 1) a positive association between an indicator of expectations and an indicator of expected economic evaluations, 2) even controlling for expectations reported in $t$, available information in $\mathrm{t}-1$, feasible to be incorporated to form expectations, is able to explain the evolution of expected evaluations, 3 ) the evidence is consistent with instances of under and over-reaction of expectations and 4) heterogeneity is not evidenced in the individuals' opinions who have different socio-demographic characteristics and political preferences. Results 1, 2 and 3 are inconsistent with the postulates of the FIRE (full-information rational expectations) models
\end{abstract}

Keywords: opinions, expectations, sentiment, macroeconomic JEL Codes: E7, E71 


\section{INTRODUCCIÓN}

Comprender cómo los individuos forman expectativas sobre variables macroeconómicas permite entender sus comportamientos y la trayectoria de agregados macroeconómicos. En este sentido, las creencias de los individuos respecto a la situación actual y al desempeño económico futuro tendrán influencia sobre su comportamiento (consumo, ahorro, inversión, stock de deuda) y, consecuentemente, sobre la dinámica macroeconómica (ver, por ejemplo, Souleles, 2004; Brown \& Taylor, 2006; Leduc \& Sill, 2010; Lanzilotta, 2015 y Gennaioli, Ma \& Shleifer, 2015).

Las opiniones de los agentes respecto a ciertos tópicos de interés, como la situación económica general del país, están determinadas por múltiples factores tales como sus preferencias, sus creencias preexistentes, la información que reciben del entorno y la forma en que procesan dicha información (ver BID, 2008). Este trabajo pretende estudiar cómo los individuos forman sus opiniones ${ }^{1} \mathrm{y}$ cómo estas varían ante el arribo de información económica de interés (ver, por ejemplo, Carroll, 2003a, 2003b; Lamla, 2008; Golub \& Jackson, 2010)². Particularmente, se pretende dar respuesta a los siguientes interrogantes: ¿existen vínculos fuertes entre opiniones e indicadores tradicionales de desempeño económico? ¿Cuál es la medida en que las expectativas reportadas proveen información sobre evaluaciones futuras? Los individuos al formular sus expectativas, ¿utilizan toda la información disponible de manera eficiente? Este trabajo, por lo tanto, pretende arrojar luz sobre los procesos de formación de opiniones.

En pos de atender el proceso de formación de opiniones, se utilizan las encuestas de opinión pública recolectada por la Corporación Latinobarómetro. A partir de estas, se obtienen las expectativas y las evaluaciones de desempeño económico de los individuos de 18 países de América Latina y el Caribe para el período 1995-2016.

A través de diversos ejercicios estadísticos se intenta caracterizar las expectativas y las evaluaciones de desempeño económico de los individuos. En particu-

1 En este trabajo, se entiende por opiniones a creencias respecto al desempeño económico futuro (expectativas) y creencias respecto al desempeño económico corriente (evaluaciones de la situación actual).

2 En este trabajo, se entiende el arribo de información económica de interés como la llegada de nuevos datos económicos. Además, este trabajo se concentra en el contenido de dichos flujos de información, sin considerar los medios a través de los cuales la información arriba a las personas. Respecto a esto último, múltiples trabajos se concentran en las fuentes de dichos flujos de información, las cuales pueden ser múltiple: medios de comunicación, televisión, redes sociales y otras formas de aprendizaje social. 
lar, se evalúa la medida en que las expectativas expresadas por los individuos, al momento de ser encuestados, contienen información sobre las evaluaciones futuras del desempeño económico que estos realizaran. Más específicamente, se proponen modelos simples de predicción de opiniones. Sobre la base de estos modelos, se analiza si la información económica preexistente al momento en que se reportan las expectativas no fue incorporada plenamente y, por tanto, contiene información adicional que contribuye a mejorar dichas expectativas. Un resultado positivo es consistente con ineficiencias en el proceso de formación de expectativas e individuos que no incorporan completamente la información disponible en sus expectativas.

Este estudio evidencia, mediante un modelo simple de predicción de evaluaciones de desempeño, tres principales resultados. En primer lugar, las expectativas formuladas por los agentes contribuyen a anticipar cómo estos evaluarán el desempeño económico. Este resultado es consistente con lo esperado. En segundo lugar, se encuentra que, al momento en que se reportan las expectativas, ciertos flujos preexistentes contienen información relevante sobre las evaluaciones futuras que no fueron incorporadas durante el proceso de formación de expectativas. Esto revela la existencia de ineficiencias en el proceso de formación de expectativas. Asociado a este último resultado, en cuarto lugar, se evidencia que existe información que no fue capturada por este proceso. Por un lado, una posible interpretación para estos resultados se asocia con la presencia de individuos que poseen recursos cognitivos limitados, quienes no incorporan toda la información disponible y, de esta manera, sub-reaccionan a flujos entrantes de información. Por otro lado, se encuentra evidencia de respuestas individuales exageradas al momento de formular sus expectativas. Este resultado es consistente con instancias de sobre-reacción ${ }^{3}$. A partir de estos resultados, se puede inferir que las expectativas se actualizan de manera parcial e incompleta ${ }^{4}$. Esta evidencia sugiere la conveniencia de ir más allá de los postulados de los modelos FIRE (full-information rational expectations) ${ }^{5}$.

Adicionalmente, se evaluó en qué medida los individuos con diferentes recursos cognitivos incorporan y procesan la información que reciben del entorno en pos de formular opiniones sobre la situación económica general. En este sentido, se agrupó a los encuestados según sus características sociodemográficas e inclinación política.

3 Diversos argumentos pueden explicar los eventos de sub-reacción y sobre-reacción. En particular, el exceso de confianza, el anclaje y la heurística de representatividad (Barberis et al., 1998), inatención (Sims, 2003), manadas (Bischi et al., 2006).

4 Este resultado es consistente con modelos en los cuales la actualización de las expectativas ocurre de manera escalonada (Mankiw \& Reis, 2002, Mankiw, Reis \& Wolfers, 2003).

5 Un análisis de estos modelos se puede encontrar en Coibion \& Gorodnickenko (2015). 
A partir de estos ejercicios se evidencia, por un lado, que los resultados hallados confirman aquellos descriptos anteriormente y, por otro lado, que no existe heterogeneidad en el proceso de formulación de opiniones económicas asociadas a la situación económica del país, ya que las ponderaciones que asignan los individuos a los diferentes flujos entrantes de información son simétricas

Este trabajo pertenece a un grupo de contribuciones que analizan expectativas y percepciones de los agentes económicos (ver, por ejemplo, Souleles, 2004; Brown \& Taylor, 2006; Leduc \& Sill, 2010; Lanzilotta, 2015 y Gennaioli et al., 2015 y Das et al., 2017). En particular este trabajo se vincula con un amplio cuerpo de la literatura que analiza en qué medida las percepciones de los agentes incorpora la información disponible y en qué medida existen diferencias entre dichas percepciones y la evolución de los indicadores objetivo (De Vries et al., 2017). Este trabajo evidencia la existencia de sesgos cuya fuente responde a la presencia de rigideces en la información (ver, por ejemplo, Coibion \& Gorodnickenko, 2015; Lamla, 2008; Mankiw \& Reis, 2002; Woodford, 2003; Sims, 2003 y Sims, 2010), inatención (Woodford, 2003, Sims, 2003 y Sims, 2010), procesos de aprendizaje social (Carroll, 2003a, 2003b; Blinder \& Krueger, 2004; Dominitz \& Manski, 2005; Lamla, 2008 y Malmendier \& Nagel, 2013), memorias imprecisas (Sims, 2003; Sims, 2010; Andrade y Le Bihan, 2013; Cavallo, Cruces \& Perez-Tuglia, 2016 y 2017) y la utilización de heurísticas (Tversky \& Kahneman, 1974). Los patrones documentados en este estudio también son consistentes con la existencia de ineficiencias en el proceso de formación de expectativa y, por tanto, la conveniencia de ir más allá de los postulados de modelos con expectativas racionales bajo información completa (FIRE). Por último, este trabajo realiza una contribución a la literatura que intenta comprender el proceso de formación de opiniones de los individuos en presencia de rigidices en la información con especial atención en países en desarrollo e incorpora técnicas de procesamiento de lenguaje natural al campo de la economía en pos de explotar fuentes de información no estructurada.

Luego de esta introducción, en la siguiente sección se describen los datos y la metodología. La sección III analiza las estadísticas descriptivas. Los resultados principales se informan en la sección IV. En la sección V, se incorpora al análisis información sociodemográfica e inclinación política de los individuos. Por último, la sección VI resume las conclusiones. 


\section{DATOS Y METODOLOGÍA}

Los datos utilizados sobre expectativas y evaluaciones referidas al desempeño económico, se obtienen a partir de encuestas a individuos. Si bien la encuesta cubre muchos tópicos, por ejemplo, las expectativas y/o percepciones de los individuos, como así también, características sociodemográficas de las familias, en esta investigación solo se tendrá en cuenta aquellas preguntas que analizan la trayectoria pasada y futura de la economía, las cuales admiten respuestas cualitativas a dichas preguntas.

Se analiza la formación de expectativas sobre la trayectoria de la performance económica y la evaluación actual de estas expectativas para 18 países de América Latina y el Caribe para el período 1995-2016. Los países bajo estudio son Argentina, Bolivia, Brasil, Chile, Colombia, Costa Rica, Ecuador, El Salvador, Guatemala, Honduras, México, Nicaragua, Panamá, Paraguay, Perú, República Dominicana, Uruguay y Venezuela.

Las encuestas son realizadas anualmente, desde el año $1995^{6}$, por la Corporación Latinobarómetro. Esta encuesta es representativa del 100\% de la población de cada uno de los países, comprendiendo áreas urbanas y rurales. A partir de estas encuestas, se construye un indicador de evaluación contemporánea y un indicador de expectativarespecto al desempeño económico. Estos indicadores son construidos utilizando las respuestas a las siguientes preguntas (las respuestas permitidas se encuentran dentro de los corchetes):

i) ¿Considera Ud. que la situación económica actual del país es mejor, igual o peor que hace doce meses? [mejor, igual, peor].

ii) ¿Y en los próximos doce meses, cree Ud. que, en general, la situación económica del país será mejor, igual o peor que ahora? [mejor, igual, peor] ${ }^{7}$.

Dichas preguntas hacen referencia a la situación económica de un país y, en particular, indagan sobre la creencia de un individuo respecto a la misma. Teniendo en que cuenta la influencia que ejercen las creencias económicas de los individuos

6 Con excepción de los años 1999, 2012 y 2014, en los cuales no se realizó dicha encuesta.

7 Ambas preguntas admitieron a partir del 2001 al 2015 las respuestas: mucho mejor, un poco mejor, igual, un poco peor, mucho peor. Por lo tanto, con el objetivo de obtener una medida que pueda ser comprable a lo largo de todo el período, dichas respuestas fueron tratadas utilizándose las categorías inicialmente propuestas por las encuestas (a saber: mejor, igual o peor), según corresponda el caso. 
sobre su comportamiento y, en última instancia, sobre la dinámica de los agregados macroeconómicos, ambas preguntas pueden ser utilizadas para analizar la evolución de la situación económica. En este sentido, la primera pregunta solicita la opinión del encuestado sobre el desempeño económico actual, mientras que la segunda indaga acerca de la perspectiva económica dentro de los próximos 12 meses. Dada la estructura de cada pregunta, adicionalmente, puede utilizarse aquella referida a las expectativas de los individuos como un pronóstico de las futuras evaluaciones de estos. Ambas preguntas admiten una respuesta que está restringida a caer en una de tres categorías: mejor, igual, peor. Respuestas favorables a ambas preguntas responden a opiniones positivas de los agentes respecto al desempeño económico.

\section{I.1. Cuantificación de las respuestas de los individuos}

Siguiendo a Souleles (2004), Lahiri \& Zhao (2015) y Lanzillota (2015) se realiza una cuantificación de las respuestas cualitativas que se desprenden de las encuestas. Dicha cuantificación se realiza computando la proporción de respuestas para cada una de las categorías. Por lo tanto, vamos a usar el código 1 para la respuesta "mejor", el código 0 para "igual" y -1 para la respuesta "peor".

Haciendo $u_{t}=N_{t}^{-1} \sum_{i=1}^{N_{t}} I\left\{y_{i t}=1\right\}$ y $d_{t}=N_{t}^{-1} \sum_{i=1}^{N_{t}} I\left\{y_{i t}=-1\right\}$ siendo estas la proporción de encuestados que han dado una respuesta codificada con "1" y “-1", respectivamente ${ }^{8}$. A partir de esto definimos:

$$
{ }_{t} I_{t+k}=u_{t}-d_{t}=N_{t}^{-1} \sum_{i=1}^{N_{t}} I\left\{y_{i t}=1\right\}-N_{t}^{-1} \sum_{i=1}^{N_{t}} I\left\{y_{i t}=-1\right\}
$$

Donde ${ }_{t} I_{t+k}$ representa un indicador de creencias, el cual captura las opiniones emitidas en el momento $t$ sobre el desempeño económico en $t+k$. Esta medida, dada la estructura de las preguntas, es un indicador de desempeño económico relativo aquel exhibido un año en el pasado. A su vez, $k$ admite solo dos valores: $k=\{0,1\}$. En primer lugar, si $k=0$ la creencia reportada se refiere al desempeño económico contemporáneo en relación con aquel exhibido un año

8 Los resultados se mantienen cuando se utilizan diferentes metodologías para agregar opiniones de los encuestados. En este sentido, se evaluó: i) utilizar el código 2 para la respuesta "mucho peor", el código -1 para "un poco peor", el número 0 para la respuesta "igual", el número 1 para "un poco mejor" y el código 2 para la respuesta "mucho mejor"; ii) utilizar los números 2, 3, 1, 4 y 5 para cada categoría de respuesta, respectivamente. 
en el pasado. En segundo lugar, si $k=1$ el indicador reporta la creencia sobre el desempeño económico en el próximo año respecto a la performance actual(o bien, reporta una creencia sobre el desempeño económico en $t+1$ respecto a la performance un año en el pasado).

\section{I.2. Medidas de flujo de información}

Este trabajo evalúa si los flujos de información están sistemáticamente asociados a cómo los individuos forman sus opiniones respecto a la actividad económica.

Los flujos de información son aproximados a través de tres variables. Por un lado, se construye un indicador que refleja información sobre cambios en la tasa de crecimiento de la economía. Este es construido utilizando los datos de PBI a precios constantes en dólares del Banco Mundial ${ }^{9}$. Por otro lado, la variable que captura la vulnerabilidad o fortaleza en el frente externo de un país está dada por el cambio en el saldo del balance de cuenta corriente como porcentaje del PBI. Estos datos también se obtuvieron de la página de datos del Banco Mundial. Por último, se construye un indicador de opiniones económicas inferidas a partir del contenido de la prensa internacional. La elección de dichas variables económicas responde a que se considera que las opiniones de los individuos sobre la performance económica no dependen solo de anuncios públicos de estadísticas económicas, sino que también dependen de información y juicios prospectivos reflejados en la prensa. Estos tres flujos de información serán desarrollados con más detalle en la siguiente subsección.

Por último, se utilizan la tasa de desempleo y la tasa de inflación para realizar un análisis de robustez, teniendo en cuenta que ambas variables también caracterizan la dinámica macroeconómica de un país. Los datos referidos a estas dos últimas variables, se obtuvieron del Banco Mundial.

\section{I.2.1. Indicador de aceleración}

El arribo de nueva información sobre la tasa de crecimiento de la economía se espera que influya en la formulación de opiniones por parte de los individuos. En este sentido, un indicador del flujo de información analizado en este trabajo, por lo tanto, está relacionado a cambios en la tasa de crecimiento económica. Siguiendo

9 https://data.worldbank.org/indicator. 
a Aromí (2018), se construye un indicador de aceleración el cual compara la tasa de crecimiento reciente con la tasa de crecimiento para un período precedente más extenso. Sea $g_{c\left[t^{\prime}, t^{\prime \prime}\right]}$ la tasa de crecimiento anual del PBI para el período que comienza en $t^{\prime}$ y finaliza en $t^{\prime \prime}$. Durante el análisis posterior, la atención va a estar puesta sobre el indicador de aceleración del crecimiento para el período $[t-k, t]$, para el caso de expectativas y para el caso de evaluación del desempeño económico. Este indicador está dado por $I_{c[t-k, t]}^{G}=g_{c[t-k, t]}-g_{c[t-s, t-k]}$. Esto es, la diferencia entre la tasa de crecimiento anual, para el período más reciente de $k$ años y la tasa de crecimiento anual para el período de $s-k$ años anteriores.

En las evaluaciones desarrolladas más adelante se utilizarán dos especificaciones para capturar información reciente e información de mediano plazo. Por un lado, el indicador de aceleración de crecimiento que captura información reciente para un país dado para el período $[t-1, t]$. Este indicador viene dado por: $I_{c[t-1, t]}^{G_{C}}=g_{c[t-1, t]}-g_{c[t-4, t-1]}$. Esto es, la diferencia entre la tasa de crecimiento anual para el período anterior de un año y la tasa de crecimiento anual para el período anterior de 3 años, para un país dado c. Por último, el indicador de aceleración que captura información de mediano plazo para un país dado para el período $[t-3, t]$ . Dicho indicador viene dado por: $I_{c[t-3, t]}^{G_{M}}=g_{c[t-3, t]}-g_{c[t-18, t-3]}$. En este caso, el indicador representa la diferencia entre la tasa de crecimiento anual, para un país dado c, entre el período anterior de 3 años y la tasa de crecimiento anual para el período anterior de 15 años.

La constitución de este tipo de indicadores permite evaluar si los individuos incorporan información referida a la performance de crecimiento de una economía en el proceso de formación de opiniones. Particularmente, se evaluará si la información referida a aceleraciones en la tasa de crecimiento explica las opiniones sobre desempeño económico. La utilización de dos indicadores permite inferir la medida en que las opiniones se basan en información más reciente o más distante.

\section{I.2.2. Indicador de vulnerabilidad externa}

El segundo indicador del flujo de información que se analiza en este trabajo corresponde al saldo del balance de cuenta corrientes como porcentaje del PBI. Este indicador está dado por $I_{c[t]}^{C C}$, esto es, el saldo que toma dicho ratio en el período . Asimismo, con el objeto de facilitar la comparación con los restantes flujos de información, se trabajará con una medida dinámica de este indicador. En este sentido, en lugar de explotar la información que brinda este indicador en niveles, se trabaja con la tasa de cambio. 
Este indicador tiene por objeto captar si los agentes tienen en cuenta, a la hora de formar sus opiniones respecto al desempeño económicos, cuáles son las condiciones externas del país. Se conjetura que el saldo del balance de cuenta corriente como porcentaje del PBI es un indicador apropiado de estas condiciones.

Si bien los saldos de cuenta corriente tomados en forma aislada no contienen información concluyente sobre el estado y las perspectivas de la economía (Heymann, 1994), esta variable es incluida considerando que contiene información sobre vulnerabilidades o fortalezas que pueden influir en el desempeño futuro de la actividad económica. Un amplio conjunto de trabajos ha estudiado la sostenibilidad del desbalance en la cuenta corriente, la reversión de la cuenta corriente y sus implicancias sobre la actividad económica (ver, por ejemplo, Heymann, 1994; Calvo, 1998; Edwards, 2004; Obstfed \& Rogoff, 2007 y Reinhart \& Reinhart, 2008). En estos trabajos se encuentra que la presencia de déficit en la cuenta corriente no necesariamente conduce a evaluaciones negativas (ver, por ejemplo, Sachs, 1981; Heymann, 1994 y Blanchard \& Giavazzi, 2002).

Teniendo en cuenta que los países de América Latina y el Caribe, a lo largo del período analizado, han presentado en promedio un déficit en la cuenta corriente del 3\% de su PBI (ver tabla 1), este indicador parece constituirse como un síntoma de fragilidad externa. Por lo tanto, es menester en este trabajo intentar captar si los agentes ignoran (o no) la dinámica de este indicador de fragilidad externa al formular opiniones sobre el desempeño económico.

\section{I.2.3. Índice del tono de las noticias en la prensa}

Teniendo en cuenta que los periódicos comunican la nueva información económica que arriba a los organismos estadísticos, se espera que los individuos se informen sobre el contenido de dicha información a partir de las noticias en la prensa. A su vez, estas noticias periodísticas pueden reflejar información y opiniones que no difunden dichos organismos estadísticos. Por lo tanto, con el objetivo de resumir este tipo de flujo de información, se construyó un índice sobre la base del contenido de la prensa mundial. Los niveles de optimismo y pesimismo van a ser aproximados capturando la frecuencia de palabras con contenido negativo en un subconjunto relevante de textos muestrales. En este apartado se propone un indicador que captura el tono en las noticias de la prensa, a partir del análisis lingüístico de textos y utilizando diccionarios especializados (Tetlock, 2007). 
Para capturar la información no estructurada distribuida por la prensa, se construyó una aproximación sobre la base de los artículos periodísticos del diario Wall Street Journal, un periódico internacional con cobertura mundial (este enfoque ha sido utilizado con éxito en diversos trabajos: Tetlock, 2007; Garcia, 2013 y Aromí, 2017). Los mismos se adquirieron a través del proveedor de medios ProQuest ${ }^{10}$. Con el fin de obtener un set de artículos consistentes, nos concentramos en la sección de economía de este periódico. Por último, se explotan los artículos periódicos desde el 3 de enero de 1995 hasta el 31 de diciembre del 2016.

Este análisis parte del texto original, el cual inicialmente es descompuesto en palabras individuales (procedimiento denominado tokenización de texto) con la posterior eliminación de "stop words", palabras comúnmente utilizadas por el idioma inglés que no poseen contenido relevante, tales como "a", "but", "how", "to"; esto último se realiza con el objetivo de poder concentrarse en las palabras más relevantes para nuestros fines, dejando de lado las palabras que aparecen con mayor frecuencia. Además, siguiendo prácticas habituales, se han eliminado del texto números y signos de puntuación y, por último, todo el texto se ha convertido a minúscula. Se construye un algoritmo, el cual computa un índice del tono como una aproximación al estado de las opiniones en la prensa. De esta forma se extrae el tono de las noticias en la prensa a partir del uso de técnicas de procesamiento de lenguaje natural.

Siguiendo a Aromí (2017), el primer paso para construir este índice involucra fragmentos del texto asociados a cada país. Con este objetivo, se creó una lista de palabras para cada país. Las palabras clave utilizadas incluyen: nombre del país, ciudad capital y gentilicio. Luego, para cada año del período muestral, se identifica el conjunto de artículos en los cuales está presente al menos una de estas palabras clave. Para cada uno de estos artículos, se selecciona la porción de texto que está lo suficientemente cerca a dichas palabras clave asociadas a los países. Específicamente, dicha selección corresponde a 50 palabras antes y 50 palabras después de la palabra clave identificada. El texto seleccionado para el país $c$ y el año $t$ es combinado formando una selección de texto $K_{c t}$. Esto concluye la etapa de extracción de texto.

Luego, para el cómputo de un indicador del tono de la información, se requiere identificar un set de palabras con contenido negativo. Siguiendo la contribución de Tetlock (2007), la lista de palabras negativas es construida usando la

10 El texto se extrajo de http://pqasb.pqarchiver.com/djreprint/. 
categoría "negativa" provista por General Inquirer, un sitio web que provee herramientas para el análisis de datos de texto ${ }^{11}$. La lista original incluye 2291 palabras. Con el fin de mejorar la precisión del índice, se expandió esta lista original incluyéndose las formas de sustantivo plural, diferentes tiempos verbales y adverbios. Este procedimiento culminó con una nueva lista de 5364 palabras.

Sea $T_{c t}$ el número de palabras en $K_{c t}$, el texto seleccionado correspondiente al año $t$ y al país $c$ y, sea $N_{c t}$ el número de veces que la palabra negativa es identificada en $K_{c t}$. Entonces, el índice del tono en las noticias está dado por $S_{c t}=N_{c t} / T_{c t}$. Por lo tanto, un mayor valor de este índice es asociado a opiniones más pesimistas mientras que valores más bajos del mismo son asociados a opiniones más optimistas. Asimismo, en pos de facilitar la interpretación de este indicador, el mismo es multiplicado por 1 con el objeto de obtener $S_{c t}=-N_{c t} / T_{c t}$, esto sugiere que mayores valores en el índice indican mayores niveles de optimismo en las opiniones de la prensa. Por último, con el objeto de facilitar la comparación con los restantes flujos de información, se trabajará con una medida dinámica de este indicador. En este sentido, en lugar de utilizar este indicador en niveles se computa el cambio de dicha variable.

\section{ESTADÍSTICAS DESCRIPTIVAS}

A partir de los datos que se exhiben en la tabla 1, se desprende que para todo el período de la muestra analizado (1995-2016), el número promedio de palabras negativas en un artículo representa el 6\% del total de palabras, es decir, un artículo típico posee un cierto nivel de pesimismo en el tono de las noticias en la prensa.

Por otro lado, se puede observar que la media de las expectativas de los individuos $\left({ }_{t} I_{t+k}\right)$ respecto a la futura performance de la economía está centrada en 0.04. Esto indica que, en promedio, la proporción de personas que cree que la economía mejorará fue levemente mayor a la proporción de personas que cree que la economía emporará. Sin embargo, la mediana de esta variable se encuentra a la izquierda de su medía, indicando la presencia de cierto nivel de asimetría en la distribución. De aquí se desprende que poco más de la mitad de los individuos posee expectativas menos optimistas respecto al futuro de la performance económica. A su vez, este indicador alcanzó su máximo en Brasil en el año 2010, que en dicho año alcanzó una tasa de crecimiento interanual del 7.5\% (su mayor alza desde 1986), luego de haber registrado una contracción moderada en el año $2009^{12}$.

11 http://www.wjh.harvard.edu/inquirer/homecat.htm

12 Según el World Economic Outlook del FMI, disponible en: https://www.imf.org/external/pubs/ft/ 
Al observar el indicador de evaluación de la situación actual de la economía por parte de los individuos $\left({ }_{t} I_{t}\right)$, se encuentra que la media es similar a la mediana. Ambas medidas son negativas, esto indica que, en promedio, es mayor la proporción de personas que cree que la situación económica durante los últimos doce meses, en general, estuvo mejor respecto a la performance actual. Este indicador alcanzó su máximo en Paraguay en el año 2008, año en el cual el PBI creció a una tasa interanual del $6.36 \%$ y cuya economía se beneficiaba del boom en la demanda internacional de commodities (soja y carne en el caso paraguayo) ${ }^{13}$. Por último, tanto las evaluaciones como el indicador de expectativas registran su mínimo en Honduras en el año 1996 (ver tabla 1).

Continuando con el análisis de estadística descriptiva de los flujos de información, como se desprende de la tabla 1, los países de la región durante el período 1995-2016 presentaron, en promedio, un déficit en cuenta corriente de 3\% de su PBI. Durante el mismo período, tanto la tasa de desempleo como la tasa de inflación, en promedio, se ubicaron en el orden del $8 \%$. Sin embargo, la tasa de desempleo presentó un máximo de 19\%, el cual fue registrado en Colombia en el año 2000, mientras que el valor más alto de la tasa de inflación lo alanzó Venezuela en el año 2015. Por último, observando la tasa de crecimiento interanual podemos concluir que si bien los países, en promedio, crecieron al $4 \%$ durante el período bajo análisis, este nivel presentó una fuerte volatilidad (desvío igual al 3\%).

Tabla 1. Estadísticas descriptivas

\begin{tabular}{cccccccc}
\hline & Media & Mediana & $\begin{array}{c}\text { Desvío } \\
\text { estándar }\end{array}$ & Q1 & Q3 & Mínimo & Máximo \\
\hline$t$ & 7082.40 & 1723.50 & 13889.17 & 604.75 & 6496.00 & 4.00 & 92529.00 \\
$n$ & 406.27 & 91.00 & 797.98 & 33.75 & 364.50 & 0.00 & 5759.00 \\
$s$ & -0.06 & -0.06 & 0.02 & -0.07 & -0.05 & -0.18 & 0.00 \\
${ }_{t} I_{t+1}$ & 0.04 & 0.01 & 0.24 & -0.14 & 0.19 & -0.74 & 0.75 \\
$t I_{t}$ & -0.17 & -0.19 & 0.27 & -0.37 & -0.00 & -0.81 & 0.50 \\
$g$ & 0.04 & 0.04 & 0.03 & 0.02 & 0.06 & -0.11 & 0.18 \\
$c c$ & -0.03 & -0.03 & 0.05 & -0.05 & -0.01 & -0.19 & 0.17 \\
$\mu$ & 0.08 & 0.07 & 0.04 & 0.05 & 0.10 & 0.01 & 0.19 \\
$\pi$ & 0.08 & 0.06 & 0.10 & 0.04 & 0.09 & -0.01 & 0.74 \\
\hline
\end{tabular}

Fuente: elaboración propia.

weo/data/WEOhistorical.xlsx

13 Según el World Economic Outlook del FMI, disponible en: https://www.imf.org/external/pubs/ft/ weo/data/WEOhistorical.xlsx 
La tabla 1 presenta las estadísticas descriptivas del total de palabras $(t)$, las palabras negativas $(n)$, el indicador del tono en las noticias de la prensa bruto $(s)$, el indicador de expectativas de los individuos $\left(\mathrm{I}_{\mathrm{t}} \mathrm{t}_{1+1}\right)$, indicador de evaluación $\left(\mathrm{t}_{\mathrm{t}}\right)$, la tasa de crecimiento interanual $(g)$, el saldo de cuenta corriente como porcentaje del PBI $(c c)$, la tasa de desempleo $(\mu)$ y la tasa de inflación $(\pi)$

\section{EVALUACIÓN DEL CONTENIDO INFORMATIVO DE LAS EXPECTATIVAS}

En esta sección, se evaluará la medida en que las expectativas reportadas por los individuos reflejan información sobre evaluaciones posteriores. Este ejercicio puede ser interpretado como una evaluación de la eficiencia del proceso de formación de expectativas. Un escenario en el que las expectativas reflejan toda la información disponible es consistente con un proceso de formación de expectativas eficiente. En cambio, un escenario en el que existen datos disponibles que proveen información adicional, es consistente con ineficiencias en las expectativas. Estas ineficiencias pueden corresponder a instancias en las que información relevante no es incorporada por los individuos durante el proceso de formación de expectativas. Esto último puede observarse en presencia de individuos que poseen recursos cognitivos limitados, quienes fallan al incorporar toda la información disponible.

Los individuos el próximo año reportarán, cuando sean encuestados, sus creencias sobre el desempeño económico contemporáneo respecto a la performance económica exhibida 12 meses en el pasado. Por lo tanto, se estiman dichas opiniones como una función de los valores rezagados de las expectativas e indicadores de la situación económica. Más específicamente, se analiza la asociación entre la evaluación contemporánea que realizan los agentes en $t+1$, con las expectativas que forman en $t$ y los flujos de información en $t-1$. La restricción al conjunto de información en $t-1$ responde al objetivo de no incorporar en el análisis información que los individuos no conocían o disponían a la hora de formular un reporte subjetivo. Por lo tanto, el modelo de datos en panel es:

$$
\begin{gathered}
{ }_{t+1} I_{t+1, c}=\alpha_{t}+\alpha_{c}+\beta_{E}{ }_{t} I_{t+1, c}+\beta_{G_{C}} I_{[t-2, t-1], c}^{G_{C}}+ \\
\beta_{G_{M}} I_{[t-4 t-1] c}^{G_{M}}+\beta_{C C} I_{[t-1] c}^{C C}+\beta_{S} I_{[t-1] c}^{S}+\mu_{[t+1] c}
\end{gathered}
$$

donde c indica el país, $\alpha_{t}$ representa el efecto fijo por período, el cual captura shocks globales que son comunes a todos los países en cada año. El coeficiente $\alpha_{c}$, 
es el efecto fijo por país, el cual captura características culturales e institucionales que difieren entre países, las cuales no varían en el tiempo. $\mu_{c[t+1]}$ es el término de error. $\beta_{G_{C}}, \beta_{G_{M}}, \beta_{C C}, \beta_{S}$, son los parámetros de interés a estimar correspondientes a cada uno de los flujos de información descriptos en la sección 3, esto es, el indicador de aceleración de crecimiento constituido para una ventana corta de información, el propio constituido que comprar períodos más distantes de tiempo, el cambio en el indicador de vulnerabilidad externa y el cambio en el indicador del tono en las noticias, respectivamente. Por último, $\beta_{E}$ corresponde al coeficiente de las expectativas respecto a la performance futura de la economía, formuladas por los agentes en t. Se espera que expectativas positivas formuladas en $t$ anticipen una evaluación futura positiva. Bajo la hipótesis de unproceso de formación de expectativas eficiente, los flujos de información no deberían ser estadísticamente significativos. Sin embargo, resultados que se alejen de esta premisa son consistentes con ineficiencias en el proceso de formación de expectativas. En este sentido, pueden tener lugar fenómenos de sub-reacción o sobre-reacción. Respecto a lo primero, se espera que el arribo de información positiva referida a cada uno de estos flujos de información genere un aumento en la evaluación esperada respecto a la performance económica corriente (coeficiente positivo y estadísticamente significativo). Respecto al segundo fenómeno, se espera que el arribo de información genere una caída en la evaluación esperada respecto a la performance económica corriente, siendo consistente con respuestas individuales exageradas a ciertos flujos de información durante el proceso de formación de expectativas (coeficiente negativo y estadísticamente significativo). Para facilitar las comparaciones de los coeficientes estimados, todas las variables explicativas han sido estandarizadas.

La tabla 2 muestra los resultados de la estimación del modelo que intenta caracterizar la relación entre la evaluación del desempeño económico futuro, los flujos de información en $t-1$ y las expectativas formuladas en respecto a la performance económica. En primer lugar, el indicador de expectativas contribuye a anticipar la evaluación esperada, siendo consistente con lo esperado. Por otro lado, a partir de la columna 1 a 5, se observa que, al evaluarse individualmente los flujos de información, controlando por las expectativas, el indicador de aceleración conformado para una ventana más reciente, el indicador de vulnerabilidad externa y el indicador de opiniones en la prensa resultan estadísticamente significativos para explicar la evolución de las evaluaciones esperadas. Sin embargo, el indicador de aceleración constituido con información más extensa parece no ser un flujo de información relevante para anticipar la evaluación esperada.

Continuando con el análisis de la tabla 2, se evidencia que las expectativas formadas por los agentes en $t$, sobre la situación económica futura, tiene una 
asociación positiva con la evaluación esperada de la performance económica. Este resultado es robusto a cambios en la especificación del modelo (ver columnas 1 a 7). Se puede observar que un incremento en un desvío estándar en las expectativas sobre la situación económica futura de los individuos, anticipa un aumento de 0.42 desviaciones estándares en la esperanza del indicador de evaluación de la performance económica en $t+1$ respecto a la performance corriente. Este resultado es consistente con lo esperado, en el sentido de que bajo ineficiencia en las expectativas no se esperaba una relación uno a uno entre las expectativas y la evaluación esperada. Asimismo, el hecho de que el coeficiente sea menor que uno evidencia la existencia de sesgos sistemáticos en las percepciones. Este resultado está en línea con la evidencia reportada por Cruces et al. (2013).

A partir de la columna 6 también se desprende que el indicador de aceleración del crecimiento constituido con información más reciente, tiene una asociación estadísticamente significativa con la evaluación esperada. Esta asociación positiva entre un indicador de crecimiento económico y un indicador que reporta una evaluación retrospectiva está en línea con la evidencia reportada para Reino Unido por De Vries et al. (2017). Por otro lado, el indicador que captura la dinámica del saldo de la cuenta corriente como porcentaje del PBI es estadísticamente significativo al 1\%. En particular, un incremento de un desvío estándar en el cambio del saldo de cuenta corriente en $t-1$ anticipa, en promedio, una caída 0.077 desviaciones estándares el indicador de la evaluación que realizaran los individuos de la performance económica en $t+1$ respecto a $t$. Adicionalmente, el indicador de opiniones en la prensa, bajo la especificación propuesta en la columna 6 , es estadísticamente significativo al 1\%. En este caso, un incremento de una desviación estándar en el indicador que captura el cambio en los niveles de optimismo de la prensa en t-1 anticipa, en promedio, una disminución de 0.052 desviaciones estándares en el indicador de evaluación retrospectiva.

A partir de los resultados expuestos en estos últimos párrafos, se desprende que la evaluación esperada puede ser explicada, a partir de esta especificación, por las expectativas formuladas por los agentes en $t \mathrm{y}$, además, por ciertos flujos de información en $t-1$. Por lo tanto, los resultados exhibidos en la tabla 2, evidencian la existencia de ineficiencias en el proceso de formación de expectativas. Por un lado, se puede inferir que las expectativas de los agentes no parecen ser un estadístico suficiente, es decir, las expectativas formadas en parecen no estar recogiendo toda la información relevante en forma adecuada, ya que existe información en $t-1$ que puede ser utilizada para mejorar los pronósticos. Por otro lado, se reporta evidencia consistente con fenómenos de sub-reacción, es decir, los agentes durante el proceso de formación de expectativas no incorporan toda la información disponible (ver, por 
ejemplo, Sims, 2003; Woodford, 2003 y Coibon \& Gorodnichenko, 2015). En particular, el indicador que captura el arribo de información referida al indicador de aceleración de mediano plazo es estadísticamente significativo y genera un aumento en la evaluación esperada. Por último, la tabla 2 evidencia fenómenos de sobre-reacción durante el proceso de formación de expectativas de los agentes. En este caso, el arribo de información asociada a noticias recientes en la prensa y la dinámica del saldo de cuenta corriente en relación al PBI genera una disminución en la evaluación esperada, indicando que los individuos extrapolaron injustificadamente información difundida por la prensa y el optimismo derivado de una mejora de la situación externa del país durante el proceso de formación de expectativas ${ }^{14}$.

Otro resultado relevante que se desprende tanto de la columna 3, 6 y 7 de la tabla 2 se refiere a que el indicador de aceleración del crecimiento constituido con información más distante, incluso cuando se lo evalúa conjuntamente, parece no ser una variable relevante para anticipar cómo los agentes realizarán sus evaluaciones sobre la situación económica.

Los resultados anteriormente mencionados se mantienen si incorporamos dos indicadores económicos que contribuyen a explicar la dinámica económica. Particularmente, estos son la tasa de inflación $(\pi)$ y la tasa de desempleo $(\mu)$. Los resultados se exponen en la columna 7 de la tabla 2 . Como puede observarse, el arribo de información en $t-1$ relacionada a estos dos indicadores parece no ser relevante para anticipar cómo evaluarán los individuos la performance económica en $t+1$. Por último, bajo esta nueva especificación el signo del indicador de opiniones en la prensa continúa siendo consistente con sobre-reacción por parte de los individuos ${ }^{15}$.

Por último, en la tabla 2 se reportan los estadísticos test-F de significatividad conjunta. Respecto a dicho estadístico, se puede inferir que todas las variables son

14 Se regresó el modelo propuesto en esta sección incorporando el indicador de vulnerabilidad externa y el indicador de tono en las noticias en la prensa en niveles. Los resultados evidencian la existencia de ineficiencias en el proceso de formación de expectativas, durante el cual los agentes parecen subreaccionar a los flujos de información derivados del saldo en la cuenta corriente y sobre-reaccionan a los flujos publicados en la prensa. Sin embargo, el indicador de tono en las noticias de la prensa no es estadísticamente significativo cuando se controla por las expectativas formuladas en t y el indicador de vulnerabilidad externa.

15 Los modelos bajo la especificación propuesta en la columna 6 y 7 , fueron analizados al excluirse los outliers o valores atípicos del indicador de evaluación esperada (excluyendo su valor mínimo y máximo, aquellos por encima del percentil 99 y por debajo del primer percentil). En todos los casos, los resultados se mantienen con leves modificaciones en las magnitudes de los coeficientes. 
estadísticamente significativas en conjunto para anticipar cómo los agentes evaluarán la performance económica, indicando que las expectativas no han incorporado adecuadamente la información disponible reciente proveniente de indicadores objetivos e indicadores que explotan información no estructurada publicada en periódicos.

\section{ASOCIACIÓN ENTRE OPINIONES, CARACTERÍSTICAS SOCIODE- MOGRÁFICAS E IDENTIFICACIÓN POLÍTICA}

Si bien los resultados exhibidos en la sección anterior permiten extraer nociones relevantes sobre el proceso de formación de opiniones de los individuos, los ejercicios estadísticos reportados no brindan información sobre cómo los agentes con diferentes recursos cognitivos incorporan y procesan la información para formular sus opiniones sobre la evolución de la situación económica general. En este sentido, el objetivo de esta sección es múltiple. Por un lado, se pretende examinar el efecto de los recursos cognitivos a partir de agrupar a los individuos según la información sociodemográfica de la encuesta Latinobarómetro. Por otro lado, se intenta identificar si existe evidencia que sea consistente con ponderaciones asimétricas de los flujos entrantes de información por parte de individuos con diferentes niveles sociodemográficos e inclinación política. Por último, esta sección permite evaluar en qué medida los patrones documentados en la sección anterior se mantienen cuando modificamos la muestra de los encuestados. Esta sección se vincula, por un lado, con la evidencia reportada por Das et al. (2017), quienes encuentran que la heterogeneidad en las expectativas macroeconómicas individuales están asociadas con su estatus socioeconómico y, por otro lado, con la evidencia hallada por Malmendier y Nagel (2013), quienes, a su vez, encuentran que los individuos, cuando forman sus expectativas macroeconómicas, asignan una ponderación mayor a información referida a realizaciones macroeconómicas que han experimentado a lo largo de su vida respecto a otra información histórica disponible. Por su parte, Brown y Taylor (2006) encuentran que las expectativas de los individuos están influenciadas por el ciclo de vida —individuos más jóvenes son más optimistas financieramente- - y el ciclo económico - los individuos son más pesimistas financieramente durante recesiones-. Por último, este apartado también se vincula con el trabajo de Huberman et al. (2018), quienes combinando datos de encuestas - utilizando la plataforma Pollfish y el portal MSN-y búsquedas en internet —utilizando Bing Search - evidencian que las expectativas económicas de los individuos se modifican según su identificación hacia el partido político ganador. 
Tabla 2. Evaluación del contenido informativo de las expectativas

\begin{tabular}{|c|c|c|c|c|c|c|c|}
\hline & (1) & (2) & (3) & (4) & (5) & (6) & (7) \\
\hline \multirow{3}{*}{$\beta_{E}$} & $0.452^{*}$ & $0.426^{*}$ & $0.457^{*}$ & $0.444^{*}$ & $0.462^{*}$ & $0.420^{*}$ & $0.460^{*}$ \\
\hline & $(0.061)$ & $(0.063)$ & $(0.062)$ & $(0.060)$ & $(0.06)$ & $(0.062)$ & $(0.087)$ \\
\hline & {$[0.00]^{*}$} & {$[0.00]^{*}$} & {$[0.00]^{*}$} & {$[0.00]^{*}$} & {$[0.00]^{*}$} & {$[0.00]^{*}$} & {$[0.00]^{*}$} \\
\hline \multirow[t]{2}{*}{$\beta_{G_{C}}$} & & $\begin{array}{c}0.113^{*} \\
(0.048)\end{array}$ & & & & $\begin{array}{c}0.113^{*} \\
(0.046)\end{array}$ & $\begin{array}{l}0.098 \\
(0.052)\end{array}$ \\
\hline & & [0.063] & & & & [0.095] & [0.095] \\
\hline \multirow[t]{2}{*}{$\beta_{G_{M}}$} & & & $\begin{array}{l}-0.04 \\
(0.068)\end{array}$ & & & $\begin{array}{l}-0.03 \\
(0.069)\end{array}$ & $\begin{array}{l}-0.05 \\
(0.084)\end{array}$ \\
\hline & & & [0.594] & & & [0.694] & {$[0.563]$} \\
\hline \multirow[t]{2}{*}{$\beta_{C C}$} & & & & $\begin{array}{c}-0.08^{*} \\
(0.019)\end{array}$ & & $\begin{array}{l}-0.077^{*} \\
(0.023)\end{array}$ & $\begin{array}{l}-0.154^{*} \\
(0.047)\end{array}$ \\
\hline & & & & {$[0.00]^{*}$} & & {$[0.003]^{*}$} & {$[0.002]^{*}$} \\
\hline \multirow[t]{2}{*}{$\beta_{S}$} & & & & & $\begin{array}{l}-0.06^{*} \\
(0.01)\end{array}$ & $\begin{array}{l}-0.052^{*} \\
(0.012)\end{array}$ & $\begin{array}{l}-0.042^{*} \\
(0.013)\end{array}$ \\
\hline & & & & & {$[0.00]^{*}$} & {$[0.00]^{*}$} & {$[0.007]^{*}$} \\
\hline \multirow[t]{2}{*}{$\mu$} & & & & & & & $\begin{array}{l}-0.051 \\
(0.032)\end{array}$ \\
\hline & & & & & & & {$[0.101]$} \\
\hline \multirow[t]{2}{*}{$\pi$} & & & & & & & $\begin{array}{l}0.028 \\
(0.038)\end{array}$ \\
\hline & & & & & & & {$[0.565]$} \\
\hline Observaciones & 222 & 222 & 222 & 222 & 222 & 222 & 188 \\
\hline Estadístico F & $51.215^{*}$ & $28.085^{*}$ & $25.752^{*}$ & $26.994^{*}$ & $28.740^{*}$ & $12.249^{*}$ & $7.883^{*}$ \\
\hline$R^{2}$ & 0.212 & 0.229 & 0.214 & 0.222 & 0.232 & 0.247 & 0.268 \\
\hline$R^{2}-A d j$ & 0.083 & 0.099 & 0.081 & 0.090 & 0.103 & 0.106 & 0.088 \\
\hline
\end{tabular}

Nota: entre paréntesis se encuentra el error estándar robusto y agrupado a nivel país y entre corchetes se encuentra el p-valor, el cual fue estimado en base a clustered errors siguiendo el método wild bootsrap (Cameron et al., 2008). ${ }^{*} \mathrm{p}<.1 ; * * \mathrm{p}<.05 ; * * * \mathrm{p}<.01$

La variable dependiente son las evaluaciones esperadas de los individuos $\left({ }_{t+1} \mathrm{I}_{\mathrm{t}+1}\right)$. Los flujos de información son capturados a través del indicador de aceleración del crecimiento constituido para una ventana corta de tiempo $\left(\beta_{G c}\right)$, el indicador de aceleración de mediano plazo $\left(\beta_{G M}\right)$, el cambio en el indicador de vulnerabilidad externa $\left(\beta_{c c}\right)$ y el cambio en el indicador del tono en las noticias de la prensa $\left(\beta_{s}\right)$. Además, las expectativas formuladas por los individuos $\left(\beta_{E}\right)$ son una variable explicativa en este modelo. Por último, son incorporadas al análisis la tasa de inflación $(\pi)$ y la tasa de desempleo $(\mu)$ como variables alternativas que captan las condiciones macroeconómicas. Para facilitar la comparación entre los coeficientes estimados, todas las variables explicativas han sido estandarizadas. 
La tabla 3 reporta los resultados del modelo especificado en la columna 6 de la tabla 2 para cada grupo de individuos según su edad, género, nivel educativo alcanzado e inclinación política. En primer lugar, se agrupa a los encuestados según su nivel educativo en tres grupos (columnas 1 a 3 en la tabla 3): i) nivel educativo bajo (NEB): analfabeto, básica incompleta y básica completa; ii) nivel educativo medio (NEM): secundaria, media y técnica incompleto y secundaria, media y técnica completo; y iii) nivel educativo alto (NEA): superior incompleto y superior completo $^{16}$. En segundo lugar, se agrupa a los individuos en dos grupos según su edad (columnas 4 y 5): i) adultos: aquellos encuestados cuya edad es mayor o igual a 30 años y ii) jóvenes: individuos con una edad menor o igual a 29 años ${ }^{17}$. En tercer lugar, se utiliza la pregunta: "En política se habla normalmente de izquierda y derecha. En una escala donde ' 1 ' es la izquierda y '10' la derecha, ¿dónde se ubicaría Ud.?”. A partir de esta consulta, se agrupa a los individuos, según su inclinación política, en (columnas 6 a 8): i) izquierda: aquellos individuos cuya respuesta se ubicó en un valor inferior a 4; ii) apolíticos: aquellos individuos cuya respuesta se ubicó entre 4 y 6 y; iii) derecha: individuos cuya respuesta se admitió un valor por encima de $6^{18}$.

A partir de los resultados exhibidos en la tabla 3 se puede apreciar que los resultados sugieren, por un lado, que no existe una heterogeneidad en el proceso de formulación de opiniones económicas asociadas a la situación económica del país ya que las ponderaciones por parte de los individuos a los diferentes flujos entrantes de información son simétricas. Por otro lado, los resultados sugieren que independientemente de los recursos cognitivos de los agentes, el proceso de formación de expectativas no parece recoger toda la información relevante en forma adecuada, ya que existe información en $t-1$ que puede ser utilizada para mejorar los pronósticos. Estos resultados evidencian la existencia de ineficiencias en el proceso de formación de expectativas. Una fuente de dicha ineficiencia se asocia a fenómenos de sub-reacción, es decir, la existencia de individuos con recursos cognitivos limitados, quienes fallan al incorporar toda la información disponible. Otra fuente se asocia a fenómenos de sobre-reacción, explicados por circunstancias en que las expectativas responden excesivamente debido a una extrapolación injustificada o respuestas indebidas a noticias entrantes producto de una ponderación excesiva de la experiencia reciente.

16 Se arriba a similares resultados si la forma de agrupación es: i) nivel educativo bajo (NEB): analfabeto y básica incompleta; ii) nivel educativo medio (NEM): básica completa, secundaria, media y técnica incompleto y secundaria, media y técnica completo, superior incompleto; y iii) nivel educativo alto (NEA): superior completo.

17 Se arriba a similares resultados si la forma de agrupación es: i) individuos con edad menor estricta a 40 años; ii) individuos cuya edad este en 40 y 60 años; y iii) individuos con edad mayor estricta a 60 años.

18 La elección de estos parámetros responde a que el primer cuartil de la variable, construida a partir de la información derivada de la pregunta sobre inclinación política, es 3 y el tercero cuartil de la misma es 7. 
Tabla 3. Evaluación del contenido informativo de las expectativas según características sociodemográficas e identificación política

\begin{tabular}{ccccccccc}
\hline & $(1)$ & $(2)$ & $(3)$ & $(4)$ & $(5)$ & $(6)$ & $(7)$ & $(8)$ \\
\hline \multirow{3}{*}{$\beta_{E}$} & NEB & NEM & NEA & Joven & Adulto & Izquierda & Apolítico & Derecha \\
\cline { 2 - 8 } & $0.372^{* * *}$ & $0.449^{* * *}$ & $0.340^{* * *}$ & $0.448^{* * *}$ & $0.403^{* * *}$ & $0.438^{* * *}$ & $0.435^{* * *}$ & $0.388^{* * *}$ \\
& $(0.064)$ & $(0.063)$ & $(0.064)$ & $(0.070)$ & $(0.060)$ & $(0.067)$ & $(0.06)$ & $(0.065)$ \\
\hline \multirow{2}{*}{$\beta_{G_{C}}$} & $0.111^{*}$ & $0.093^{*}$ & $0.131^{* * *}$ & $0.110^{* *}$ & $0.112^{*}$ & 0.062 & $0.127^{* *}$ & 0.083 \\
& $(0.052)$ & $(0.050)$ & $(0.042)$ & $(0.045)$ & $(0.052)$ & $(0.045)$ & $(0.044)$ & $(0.056)$ \\
$\beta_{G_{M}}$ & 0.006 & -0.052 & -0.036 & -0.022 & -0.034 & -0.009 & -0.045 & -0.048 \\
& $(0.069)$ & $(0.069)$ & $(0.069)$ & $(0.068)$ & $(0.069)$ & $(0.044)$ & $(0.063)$ & $(0.095)$ \\
$\beta_{C C}$ & $-0.079^{* * *}$ & $-0.054^{* *}$ & $-0.110^{* * *}$ & $-0.078^{* * *}$ & $-0.074^{* * *}$ & $-0.05^{* * *}$ & $-0.078^{* * *}$ & $-0.07^{* *}$ \\
& $(0.024)$ & $(0.023)$ & $(0.025)$ & $(0.026)$ & $(0.023)$ & $(0.022)$ & $(0.020)$ & $(0.028)$ \\
$\beta_{S}$ & $-0.053^{* * *}$ & $-0.054^{* * *}$ & $-0.150^{* * *}$ & $-0.052^{* * *}$ & $-0.053^{* * *}$ & $-0.054^{* * *}$ & $-0.043^{* *}$ & $-0.056^{* * *}$ \\
& $(0.013)$ & $(0.011)$ & $(0.014)$ & $(0.013)$ & $(0.012)$ & $(0.012)$ & $(0.010)$ & $(0.015)$ \\
\hline \multirow{2}{*}{ Obs. } & 222 & 222 & 222 & 222 & 222 & 222 & 222 & 222 \\
$R^{2}$ & 0.213 & 0.251 & 0.194 & 0.255 & 0.236 & 0.272 & 0.254 & 0.192 \\
\hline
\end{tabular}

Nota: entre paréntesis se encuentra el error estándar robusto y agrupado a nivel país y el p-valor fue estimado sobre la base de clustered errors siguiendo el método wild bootsrap (Cameron et al., 2008). ${ }^{*} \mathrm{p}<.1 ; * * \mathrm{p}<.05 ; * * * \mathrm{p}<.01$

La variable dependiente son las evaluaciones esperadas de los individuos $\left({ }_{t+1} \mathrm{I}_{\mathrm{t}+1}\right)$. Los flujos de información son capturados a través del indicador de aceleración del crecimiento constituido para una ventana corta de tiempo $\left(\beta_{G c}\right)$, el indicador de aceleración de mediano plazo $\left(\beta_{G M}\right)$, el cambio en el indicador de vulnerabilidad externa $\left(\beta_{c c}\right)$ y el cambio en el indicador del tono en las noticias de la prensa $\left(\beta_{s}\right)$. Además, las expectativas formuladas por los individuos $\left(\beta_{E}\right)$ son una variable explicativa en este modelo. Se agrupó a los encuestados según sus características sociodemográficas e identificación política

\section{CONCLUSIONES}

Comprender cómo los individuos forman sus opiniones respecto al desempeño económico actual y futuro puede ayudar a entender cómo estos se comportan, cómo toman decisiones y cómo influyen estas opiniones, en última instancia, en los agregados macroeconómicos y la dinámica económica. Además, desde el punto de vista de los hacedores de política económica, dicha información puede ser útil para interpretar eventos macroeconómicos de interés y anticipar el impacto que tendrán ciertas medidas de política. 
En este trabajo se estudia la asociación entre las opiniones de los individuos respecto al desempeño económico y flujos de información construidos a partir de indicadores económicos de interés. Las opiniones provienen de encuestas de opinión pública realizadas a individuos de 18 países de América Latina y el Caribe para el período 1995-2016. Dichas encuestas son recolectadas por la ONG Latinobarómetro.

En particular, se propuso un modelo simple de predicción de las evaluaciones de desempeño por parte de los individuos. A partir de este modelo se encuentra que tanto las expectativas formuladas por los individuos en como ciertos flujos de información en contribuyen a anticipar el valor futuro de las evaluaciones de desempeño económico contemporáneas. Estos resultados son consistente con ineficiencias en el proceso de formación de expectativas. En otras palabras, se observa que existe información relevante, al momento en que se reportan las expectativas, que no fue incorporada en forma adecuada. En este sentido, este trabajo evidencia instancias de sub-reacción asociadas a la demora en la percepción de información disponible e instancias de sobre-reacción vinculadas a respuestas exageradas por parte de los individuos. El primer fenómeno responde a la presencia de individuos que poseen recursos cognitivos limitados, quienes fallan en incorporar toda la información disponible y sub-reaccionan a ciertos flujos de información. Con respecto al segundo, se evidencia una extrapolación injustificada que se deriva de una ponderación excesiva a noticias de la prensa y al optimismo generado por la dinámica de un indicador que captura el frente externo del país.

En tercer lugar, este modelo simple de predicción se extiende para incorporar información sobre recursos cognitivos de los individuos e información asociada a su inclinación política. A partir de estos ejercicios, los resultados sugieren que los patrones documentados en este trabajo se mantienen y no se evidencia heterogeneidad en el proceso de formulación de opiniones económicas asociadas a la situación económica del país, ya que las ponderaciones que asignan los individuos a los diferentes flujos entrantes de información son simétricas

Las direcciones en las que se puede extender este trabajo son múltiples. Por un lado, una forma de extensión involucra incorporar encuestas cuya frecuencia sea más elevada o bien encuestas con muestras más extensas en pos de mejorar la precisión de las mediciones. Por otro lado, se podría desarrollar medidas más precisas de flujos de información o bien incorporar otras (por ejemplo, contenidos difundidos en otros medios de comunicación). Otra alternativa se relaciona con evaluar la capacidad predictiva del indicador de expectativas, construido a partir de opiniones, sobre la evolución del PBI. 


\section{REFERENCIAS BIBLIOGRÁFICAS}

Andrade, P. \& Le Bihan, H. (2013). Inattentive professional forecasters. Journal of Monetary Economics, 60(8), 967-982.

Aromí, J. D. (2017). Conventional Views and Asset Prices: What to Expect after Times of Extreme Opinions? Journal of Applied Economics, 20(1), 49-73.

Aromí, J. D. (2018). GDP growth forecasts and information flows: Is there evidence of overreactions?. International Finance, 21 (2), 122-139.

Barberis, N., Shleifer, A., \& Vishny, R. (1998). A model of investor sentiment. Journal of financial economics, 49(3), 307-343.

BID (2008). ¿Es la gente la que elige? La importancia de las opiniones en el proceso de formulación de políticas. En E. Lora (coord.) Calidad de vida: más allá de los hechos (Cap. 9, pp.229-248). Washington DC: Fondo de Cultura Económica. Recuperado de https://publications.iadb.org/publications/spanish/document/Calidad-de-vida-M\%C3\%A1s-all\%C3\%A1-de-los-hechos. pdf

Bischi, G. I., Gallegati, M., Gardini, L., Leombruni, R., \& Palestrini, A. (2006). Herd behavior and nonfundamental asset price fluctuations in financial markets. Macroeconomic Dynamics, 10(4), 502-528.

Blanchard, O., \& Giavazzi, F. (2002). Current account deficits in the euro area: the end of the Feldstein-Horioka puzzle?. Brookings papers on economic activity, 33(2), 147-209.

Blinder, A. \& Krueger, A. (2004). What Does the Public Know about Economic Policy, and How Does It Know It? National Bureau of Economic Research, (NBER Working Paper No. 10787).

Brown, S. \& Taylor, K. (2006). Financial Expectations, Consumption and Saving: A Microeconomic Analysis. Fiscal Studies, 27(3), 313-338.

Calvo, G. A. (1998). Çapital ows and capital market crises: The Simple Economics of Sudden Stops. Journal of Applied Economics, 1(1), 35-54.

Cameron, A. C., Gelbach, J. B., \& Miller, D. L. (2008). Bootstrap-based improvements for inference with clustered errors. The Review of Economics and Statistics, 90(3), 414-427.

Carroll, C. (2003a). Macroeconomic Expectations of Households and Professional Forecasters. Quarterly Journal of Economics, 118(1), 269-298.

Carroll, C. (2003b). The Epidemiology of Macroeconomic Expectations. Quarterly Journal of Economics, 118(1), 269-298.

Cavallo, A., Cruces, G. \& Perez-Tuglia, R. (2016). Learning from Potentially Biased Statistics. Brookings Papers on Economic Activity, 46(1), 59-108. 
Cavallo, A., Cruces, G. \& Perez-Tuglia, R. (2017). Inflation Expectations, Learning, and Supermarket Prices: Evidence from Survey Experiments. American Economic Journal: Macroeconomics, 9(3), 1-35.

Coibion, O. \& Gorodnichenko, Y. (2015). Information rigidity and the expectations formation process: A simple framework and new facts. The American Economic Review, 105(8), 2644-2678.

Cruces, G, Perez-Truglia, R. \& Tetaz, M. (2013). Biased perceptions of income distribution and preferences for redistribution: Evidence from a survey experiment. Journal of Public Economics, 98(C), 100-112.

Das, S., Kuhnen, C. \& Nagel, S. (2017). Socioeconomic status and macroeconomic expectations. National Bureau of Economic Research, (NBER Working Paper No. 24045).

De Vries, C. E., Hobolt, S. B., \& Tilley, J. (2018). Facing up to the facts: What causes economic perceptions? Electoral Studies, 51, 115-122. Recuperado de https://doi.org/10.1016/j.electstud.2017.09.006

Dominitz, J. \& Manski, C. (2004). How Should We Measure Consumer Confidence. Journal of Economic Perspectives, 18(2), 51-66.

Edwards, S. (2004). Thirty Years of Current Account Imbalances, Current Account Reversals, and Sudden Stops. IMF Staff Papers, 51(s1), 1-49.

Garcia, D. (2013). Sentiment during recessions. The Journal of Finance, 68(3), 1267-1300.

Gennaioli, N., Ma, Y., \& Shleifer, A. (2015). Expectatios and Investment. (NBER Working Paper No. 21260). Cambridge, MA: National Bureau of Economic Research. Recuperado de http://www.nber.org/papers/w21260

Golub B. \& Jackson, O. M. (2010). Naïve Learning in Social Networks and the Wisdom of Crowds, American Economic Journal: Microeconomics, 2(1), 112-149.

Heymann, D. (1994). Sobre la interpretación de la cuenta corriente. Economía Mexicana. Nueva Época, 3(1), 31-59.

Huberman, G., Konitzer, T., Krupenkin, M., Rothschild, D. \& Hill, S. (2018). Economic Expectations, Voting, and Economic Decisions around Elections. AEA Papers and Proceedings, 108, 597-602.

Lahiri, K. \& Zhao, Y. (2015). Quantifying survey expectations: A critical review and generalization of the Carlson-Parkin method. International Journal of Forecasting, 31 (1), 51-62.

Lamla, Michael J. \& Sarah M. Lein. (2008). The Role of Media for Consumers' Inflation Expectation Formation. (KOF Working Paper No. 201). Zurich: Swiss Economic Institute. Recuperado de https://doi.org/10.3929/ethza-005640674 
Lanzilotta Mernies, B. (2015). Expectativas empresariales: consecuencias en el crecimiento en Uruguay. Cuadernos de Economía, 34(65), 423-442.

Leduc, S. \& Sill, K. (2010). Expectations and economic fluctuations: and analysis using survey data. (Working Paper No. 10-6). Philadelphia: Federal Reserve Bank of Philadelphia.

Malmendier, U. \& Nagel, S. (2013). Learning from Ination Experiences. The Quarterly Journal of Economics, 131(1), 53-87.

Mankiw, N. \& R. Reis (2002). Sticky Information versus Sticky Prices: A Proposal to Replace the New Keynesian Phillips Curve. The Quarterly Journal of Economics, 117(4), 1295-1328.

Mankiw, N., Reis, R. \& Wolfers, J. (2003). Disagreement about Inflation Expectations. (NBER Working Paper No. 9796).

Recuperado de https://www.nber.org/papers/w9796

Obstfeld, M., \& Rogoff, K. (2007). The unsustainable US current account position revisited. In G7 current account imbalances: Sustainability and adjustment (pp. 339-376). Cambridge, MA: National Bureau of Economic Research.

Reinhart, C. M. \& Reinhart, V. R. (2008). Capital flow bonanzas: an encompassing view of the past and present. (NBER Working Paper No.14321).

Recuperado de https://www.nber.org/papers/w14321

Sachs, J. (1981). The Current Account and Macroeconomic Adjustment in the 1970s. Brookings Papers on Economic Activity, 1(1), 201-268.

Sims, C. (2003). Implications of rational inattention. Journal of Monetary Economics, 50(3), 665-690.

Sims, C. (2010). Rational inattention and monetary economics (Cap. 4, pp.155181). In B. M. Friedman, \& F. H. Hahn (Eds.) Handbook of Monetary Policy, vol. 3. Elsevier. Recuperado de https://EconPapers.repec.org/RePEc:eee:monchp:3-04

Souleles, S. Nicholas (2004). Expectations, Heterogeneous Forecast Errors, and Consumption: Micro Evidence from the Michigan Consumer Sentiment Surveys. Journal of Money, Credit and Banking, 36(1), 39-72.

Tetlock, Paul, (2007). "Giving content to investor sentiment: The role of media in the stock market", Journal of Finance, 1139-68.

Tversky, A. y Kahneman, D (1974). Judgment under Uncertainty: Heuristics and Biases. Science, 185(4157), 1124-31.

Woodford, M. (2003). Imperfect common knowledge and the effects of monetary policy. En P. Aghion, R. Frydman, J. Stiglitz, \& M. Woodford, M. Knowledge, Information, and Expectations in Modern Macroeconomics: In Honor of Edmund S. Phelps, (pp. 25-58). Princeton: Princeton University Press. 
(C) 2021 por los autores; licencia no exclusiva otorgada a la revista Estudios económicos. Este artículo es de acceso abierto y distribuido bajo los términos y condiciones de una licencia Atribución-No Comercial 4.0 Internacional (CC BY-NC 4.0) de Creative Commons. Para ver una copia de esta licencia, visite http://creativecommons.org/licenses/by-nc/4.0 